\title{
NOTA SOBRE PORTULACA CALIFORNICA LEGRAND (PORTULACACEAE), UNA ESPECIE ENDÉMICA POCO CONOCIDA DE BAJA CALIFORNIA SUR, MÉXICO*
}

\author{
Gilberto OCAmpo $^{1,2}$ y J. Travis Columbus ${ }^{2}$ \\ ${ }^{1}$ Instituto de Ecología, A.C., Centro Regional del Bajío, Apdo. postal 386, 61600 \\ Pátzcuaro, Michoacán, México. \\ ${ }^{2}$ Rancho Santa Ana Botanic Garden, 1500 North College Avenue, \\ Claremont, California, 91711 E.U.A. gilberto.ocampo@cgu.edu
}

\section{RESUMEN}

Portulaca californica Legrand es una de las cinco especies del género que se conocen de la península de Baja California y a pesar de que se trata de un taxon endémico al estado de Baja California Sur, se sabe poco él. Este trabajo aporta datos acerca de la especie, resultado del trabajo de campo y de la revisión de ejemplares de herbario. Se trata de plantas perennes, postradas a decumbentes, que se distinguen por tener hojas imbricadas especialmente en plantas jóvenes y en los ápices florales de individuos maduros, así como por sus flores de color magenta, cápsula con opérculo campanulado y semillas de color gris brillante. La especie se distribuye en la proximidad de la franja costera en ambos lados de la porción meridional de la península, donde la creciente expansión del desarrollo turístico representa un peligro para su subsistencia.

Palabras clave: Baja California Sur, México, Portulaca, Portulacaceae.

\section{ABSTRACT}

Portulaca californica is one of five species in the genus distributed on the Baja California peninsula and is endemic to the state of Baja California Sur. Little is known about its distribution and biology. We studied the species in the field and herbarium to improve

\footnotetext{
* Trabajo realizado con el apoyo financiero de Rancho Santa Ana Botanic Garden, de la Fundación Prywer, del University Club of Claremont, del Instituto de Ecología, A.C. y del Consejo Nacional de Ciencia y Tecnología.
} 
upon the limited knowledge. It is a perennial plant with stems prostrate to decumbent, leaves imbricate in young individuals and at branch tips in mature plants, flowers magenta, the lid of the capsule campanulate, and seeds shiny grey. The species is distributed along both coasts of the southern part of the peninsula, where accelerated development for tourism is a threat to the survival of the species.

Key words: Baja California Sur, México, Portulaca, Portulacaceae.

Portulaca californica Legrand (Portulacaceae) es una de las especies del género que junto con $P$. halimoides L., $P$. oleracea L., $P$. suffrutescens Engelm. y $P$. umbraticola Kunth subsp. lanceolata (Engelm.) J. F. Matthews \& Ketron, se distribuyen en la península de Baja California. A pesar de que se trata de un taxon relativamente bien distribuido en la parte meridional de la península, éste no se incluye en los trabajos más importantes sobre la flora de la región (e.g., Shreve y Wiggins, 1964; Wiggins, 1980), ni en un estudio reciente sobre los endemismos bajacalifornianos (Riemann y Ezcurra, 2007); únicamente Legrand $(1952,1962)$ y el Programa de Manejo del Área de Protección de la Flora y Fauna de las Islas del Golfo de California (Anónimo, 2000) registran esta especie como elemento de la flora peninsular. No obstante que este último trabajo versa sobre la parte insular de Baja California, no hace mención específica de la distribución de la especie en el sistema de islas.

La presente contribución muestra datos acerca del taxon, incluyendo su distribución, obtenidos a partir de trabajo de campo y de la revisión de las colecciones MEXU, MVM, RSA-POM y SD. Los ejemplares del herbario regional HCIB no estuvieron disponibles, pero la información de las etiquetas de colecta fue amablemente proporcionada por su curador.

Portulaca californica se encuentra distribuida en ambas costas de la porción meridional de la Península (Fig. 1), siendo la zona de la Laguna San Ignacio el punto más septentrional de su área conocida. Hasta el momento $P$. californica sólo se ha registrado de las islas de Las Ánimas, San Ildefonso y San José, pero es probable que se encuentre mejor distribuida a través del sistema insular del Golfo de California. Esta circunstancia podría sugerir la posible presencia del taxon en las costas de Sinaloa y/o Sonora, pero hasta la fecha la revisión de especímenes, así como la información proporcionada por los herbarios UAS y USON, no lo han confirmado.

Las plantas de esta especie son perennes, con raíces un tanto suberosas, de las cuales brotan tallos postrados o decumbentes, de (5)10 a $40 \mathrm{~cm}$ de largo. Las hojas son alternas, suculentas, dispuestas en internodos por lo general muy cortos, produ- 


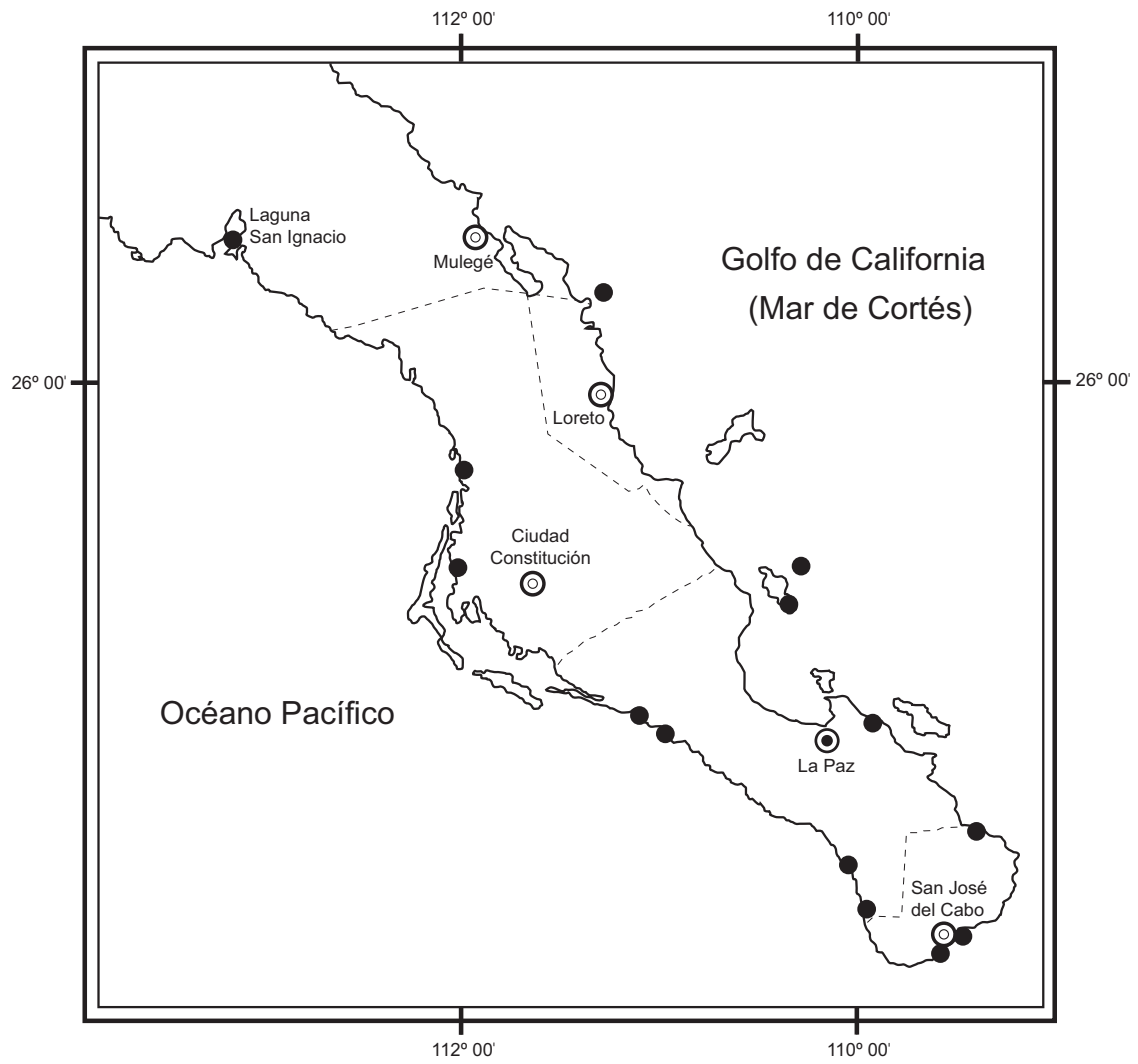

Fig. 1. Distribución conocida de P. californica en el estado de Baja California Sur.

ciendo así un patrón imbricado, sobre todo en plantas en desarrollo y en los ápices florales (Fig. 2A), con escasos pelos axilares; la lámina tiene forma oblongo-elíptica a oblongo-espatulada, con el ápice obtuso. La flor es de color magenta (Fig. 2B), de alrededor de $5 \mathrm{~mm}$ de diámetro, con 4 a 9 estambres. La cápsula mide ca. $2.5 \mathrm{~mm}$ de largo y cuenta con un opérculo de forma campanulada; contiene numerosas semillas reniformes, de color gris brillante, de aprox. $0.5 \mathrm{~mm}$ de largo, con la superficie de la testa cubierta de tubérculos cortos, casi planos, estelulados en su base (Fig. 2C). Los ejemplares se han observado en floración de septiembre a noviembre y en fruto de septiembre a mayo. Es común encontrar especímenes de la especie identificados como $P$. suffrutescens, la cual se diferencia de $P$. californica por tener tallos erectos, hojas lineales, flores de color amarillo con más de 15 estambres y por tener el opérculo de la cápsula de forma semiesférica. 

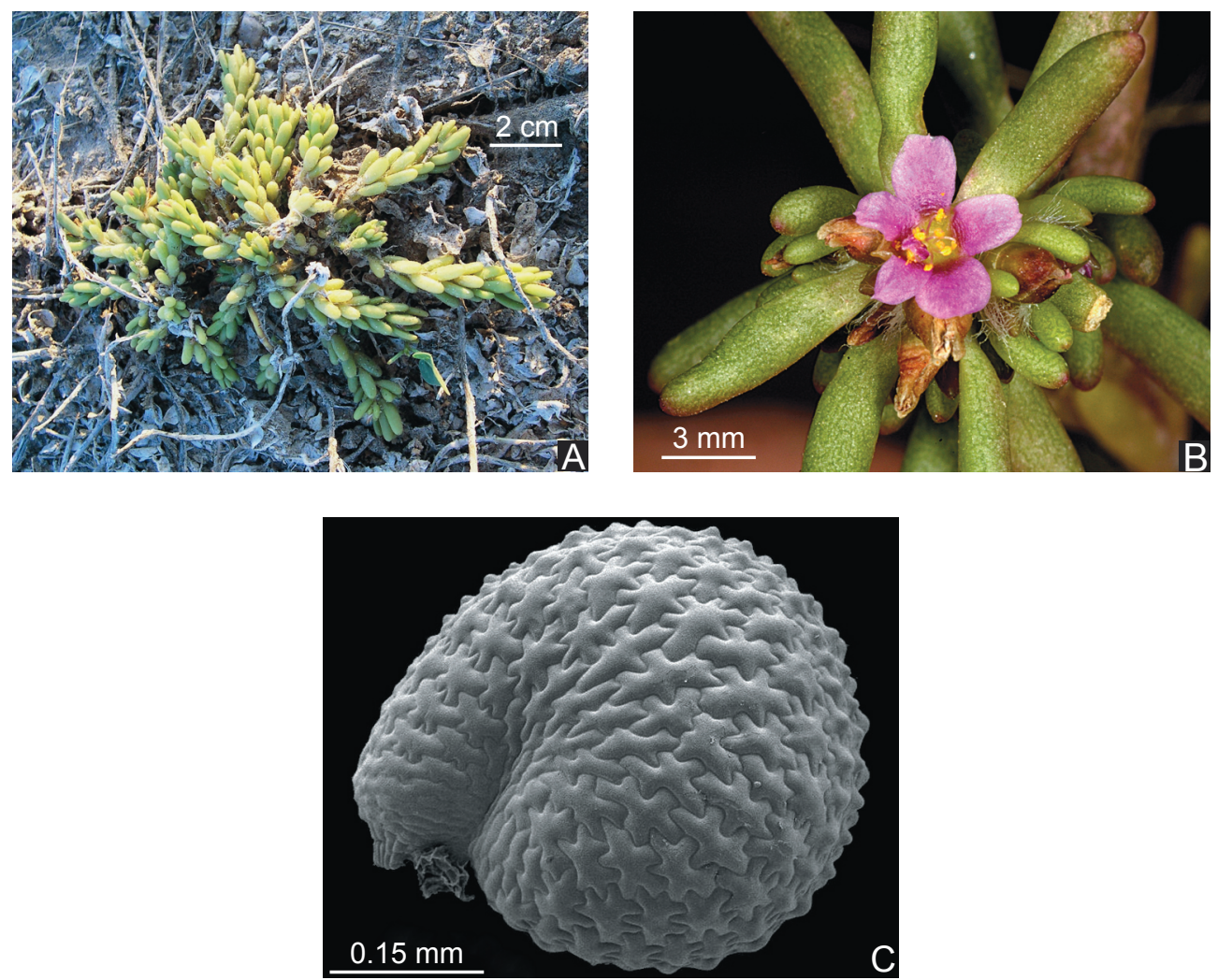

Fig. 2. Portulaca californica. A. aspecto general de la planta; B. flor y cápsulas inmaduras cubiertas por el cáliz; C. imagen de una semilla vista a través del microscopio electrónico de barrido (G. Ocampo y J. T. Columbus 1529).

El taxon se encuentra principalmente cerca de dunas costeras estables o en suelos arenosos adyacentes a las costas con vegetación de matorral sarcocaule, preferentemente a sotavento, pero también en lugares expuestos directamente a la brisa del mar. En general, la especie se halla en terrenos abiertos, planos o ligeramente inclinados. Entre las especies con las que $P$. californica se encuentra asociada frecuentemente tenemos a Boerhavia sp., Euphorbia spp., Jouvea pilosa (J. Presl) Scribn., Pectis sp., Trianthema portulacastrum L.

Debido a que la planta está distribuida en un amplio sector de la mitad sur de la península (ver Apéndice) aunado al hecho de que en los lugares en los que se encuentra puede ser abundante localmente, por el momento no se le considera dentro de alguna categoría de riesgo. No obstante, debido al ritmo acelerado de crecimiento y urbanización que experimenta la región, las poblaciones de $P$. californica se en- 
cuentran potencialmente amenazadas por la destrucción de los habitats costeros con fines de desarrollo turístico.

Los análisis filogenéticos preliminares del marcador molecular de núcleo ITS (Ocampo y Columbus, inédito), muestran a P. californica como miembro de un clado compuesto por especies norteamericanas, teniendo a $P$. suffrutescens como especie hermana. Esta última se distribuye en el norte de México (incluyendo Baja California Sur) y sur de E.U.A., en altitudes mayores a (200) $600 \mathrm{~m}$, por lo que hasta este momento, y en espera de observar la filogenia que resulte del análisis de otros marcadores moleculares, se especula que después de haberse introducido a la península, alguna de sus poblaciones se diferenció y dio lugar a $P$. californica, la cual se estableció en ambientes costeros.

Clave para las especies de Portulaca de la península de Baja California.

Clave para ejemplares con flor

1 Pétalos de color magenta P. californica Legrand

1 Pétalos de color amarillo.

2 Pelos de las axilas foliares ausentes o de hasta $1 \mathrm{~mm}$ de largo; hojas aplanadas, lanceoladas a oblanceoladas u obovadas a espatuladas, generalmente de más de $4 \mathrm{~mm}$ de ancho.

3 Plantas por lo general erectas; hojas lanceoladas a oblanceoladas u obovadas, ápice generalmente agudo, en ocasiones redondeado; ápice de los pétalos a menudo rojizo

P. umbraticola Kunth subsp. lanceolata (Engelm.) J. F. Matthews \& Ketron

3 Plantas por lo general postradas; hojas obovadas a espatuladas, ápice obtuso a redondeado; pétalos completamente amarillos P. oleracea L.

2 Pelos de las axilas foliares presentes, de más de $3 \mathrm{~mm}$ de largo; hojas rollizas, lineales a oblongas, generalmente de hasta $2 \mathrm{~mm}$ de ancho.

4 Plantas perennes con raíces carnosas, fasciculadas; tallos de más de $10 \mathrm{~cm}$ de largo, erectos P. suffrutescens Engelm.

4 Plantas anuales; tallos de hasta $8 \mathrm{~cm}$ de largo (en muy raras ocasiones más largos), postrados a decumbentes P. halimoides L.

Clave para ejemplares con fruto

1 Pelos de las axilas foliares ausentes o de hasta $1 \mathrm{~mm}$ de largo; hojas aplanadas, lanceoladas a oblanceoladas u obovadas a espatuladas, generalmente de más de $4 \mathrm{~mm}$ de ancho. 
2 Plantas por lo general postradas; opérculo de la cápsula campanulado, cubierto por un cáliz persistente; semillas de color negro P. oleracea L.

2 Plantas por lo general erectas; opérculo de la cápsula deprimido hasta casi plano, no cubierto por un cáliz persistente; línea de dehiscencia con un ala membranosa; semillas de color gris

P. umbraticola Kunth subsp. lanceolata (Engelm.) J. F. Matthews \& Ketron

1 Pelos de las axilas foliares presentes, de más de $3 \mathrm{~mm}$ de largo; hojas lineales a oblongas u oblongo-elípticas, de hasta $3 \mathrm{~mm}$ de ancho.

3 Plantas anuales; tallos de hasta $8 \mathrm{~cm}$ de largo (en muy raras ocasiones más largos) P. halimoides L.

3 Plantas perennes; tallos un tanto suberosos en su base, de más de $10 \mathrm{~cm}$ de largo.

4 Raíces carnosas, fasciculadas; tallos erectos; hojas lineales; opérculo de la cápsula hemisférico ...................................... P. suffrutescens Engelm.

4 Raíces pivotantes; tallos postrados a decumbentes; hojas oblongo-elípticas; opérculo de la cápsula campanulado P. californica Legrand

\section{AGRADECIMIENTOS}

Deseamos agradecer a Adrián Beltrán (UAS), José Luis León de la Luz (HCIB), Rosalinda Medina-Lemos (MEXU) y Jesús Sánchez-Escalante (USON) por su ayuda en la obtención de información de material de herbario, así como a Héctor Osorio (MVM), Jon Rebman (SD) y Sula Vanderplank (RSA-POM) por las facilidades otorgadas para revisar las colecciones que están a su cargo. Asimismo se agradece a Patricia Mayoral por la revisión del presente trabajo.

\section{LITERATURA CITADA}

Anónimo. 2000. Programa de manejo del área de protección de la flora y fauna de las islas del Golfo de California. Secretaría del Medio Ambiente, Recursos Naturales y Pesca. Comisión Nacional de Áreas Naturales Protegidas. México, D.F. 262 pp.

Legrand, D. 1952. Algunas especies nuevas de Portulaca. Comun. Bot. Mus. Hist. Nat. Montevideo 2(22): 2.

Legrand, D. 1962. Las especies americanas de Portulaca. An. Mus. Hist. Nat. Montevideo, 2a. ser. 7(3): 1-147.

Riemann, H. y E. Ezcurra. 2007. Endemic regions of the vascular flora of the peninsula of Baja California, Mexico. J. Veg. Sci. 18: 327-336. 
Shreve, F. e I. L. Wiggins. 1964. Vegetation and flora of the Sonoran Desert. 2 vols. Stanford University Press. Stanford, California. $1740 \mathrm{pp}$.

Wiggins, I. L. 1980. Flora of Baja California. Stanford University Press. Stanford, California. $1025 \mathrm{pp}$.

Recibido en agosto de 2007.

Aceptado en enero de 2008. 


\section{APÉNDICE}

Ejemplares de Portulaca californica revisados y/o registrados.

MÉXICO. Baja California Sur: San Ignacio Lagoon, E side, on the trail to the "cardons", municipio de Mulegé, 21.II.1977, C. Davidson 5476 (RSA-POM!); SE shore of San Ignacio

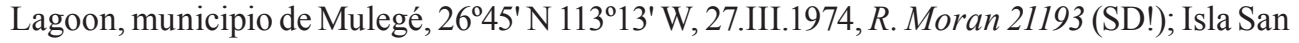
Ildefonso, NW corner, municipio de Mulegé, 26.6 N 111.4' W, 21.III.1971, J. R. Hastings 71112 (SD!); near S end of Isla San Ildefonso, SW of Mulegé, municipio de Mulegé, 15.IV.1963, I. L. Wiggins y D. B. Wiggins 18259 (MEXU!); Ildefonso Island, top near $\mathrm{S}$ end, municipio

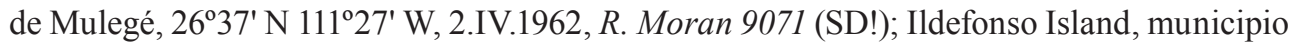
de Mulegé, 26 $6^{\circ} 37.5^{\prime} \mathrm{N} 111^{\circ} 26.5^{\prime} \mathrm{W}, 14 . \mathrm{V} .1952$, R. Moran 4138 (MEXU!, RSA-POM!, SD!); Boca de las Ánimas, $\mathrm{N}$ end of Magdalena Bay, municipio de Comondú, 25⒋ N $112^{\circ} 03^{\prime} \mathrm{W}$, 29.IV.1972, P. J. Mudie 838 (SD!); La Soledad, estero al S del poblado Puerto Adolfo Mateos, en la parte norcentral del estero, al SW del tanque grande de agua, al $\mathrm{S}$ de la gasolinería que se encuentra a la entrada del pueblo, municipio de Comondú, $25^{\circ} 11.330^{\prime} \mathrm{N} 112^{\circ} 05.907^{\prime} \mathrm{W}$, 27.X.2005, G. Ocampo y J. T. Columbus 1497 (RSA-POM!); Isla Las Ánimas, E of Isla San José, municipio de La Paz, 12.IV.1962, I. L. Wiggins 17676 (MEXU!); Las Ánimas Rock, municipio de La Paz, $25^{\circ} 06.5^{\prime}$ N $110^{\circ} 31.5^{\prime}$ W, 12.IV.1962, R. Moran 9435 (SD!); Isla San José, SW side, municipio de La Paz, 245''ㄹ" N 110³7'35" W, 24.IX.1996, J. Rebman et al. 3593 (HCIB,

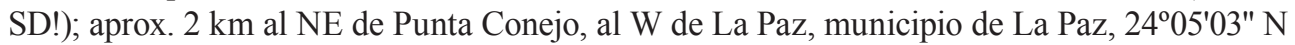
$110^{\circ} 00^{\prime} 40^{\prime \prime}$ W, 27.X.2005, G. Ocampo y J. T. Columbus 1499 (RSA-POM!); about 1/4 mi. SW of Guadalupe, municipio de La Paz, 29.IV.1931, I. L. Wiggins 5547 (holotipo: CAS; isotipos: MVM!, NY, UC); vicinity of Rancho Las Cruces, especially large granite wash

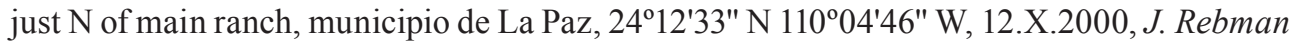
6993 (HCIB, SD!); in sandy soil along margin of loading strip at Las Cruces, on Gulf coast E of La Paz, municipio de La Paz, 17.XII.1958, I. L. Wiggins 14390 (MEXU!); N of Todos

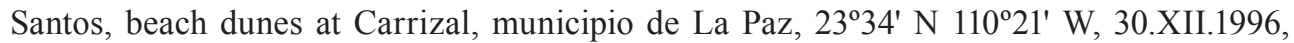
B. Vinton 35 (HCIB, SD!); al NW del poblado Todos Santos, sobre una brecha que corre

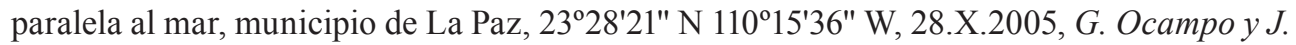
T. Columbus 1506 (RSA-POM!); orilla de pequeña bahía en el Hotel Las Pozas, en el poblado

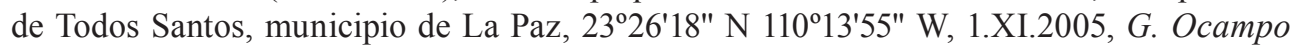
y J. T. Columbus 1529 (RSA-POM!); al S de la playa Los Cerritos, al N de Rancho Nuevo, al S de Todos Santos, sobre la carretera a Cabo San Lucas, municipio de La Paz, $23^{\circ} 17^{\prime} 08^{\prime \prime} \mathrm{N}$ $110^{\circ} 09^{\prime 29}$ "W, 29.X.2005, G. Ocampo y J. T. Columbus 1507 (RSA-POM!); playa al NE de La Ribera, municipio de Los Cabos, 2335'48" N 109³4'32" W, 29.X.2005, G. Ocampo y J. T. Columbus 1514 (RSA-POM!); playa Costa Azul, entre los hoteles Crown Plaza y Posada Real, en la zona hotelera de San José del Cabo, municipio de Los Cabos, 2302'37" N 10941'52" W, 29.X.2005, G. Ocampo y J. T. Columbus 1511 (RSA-POM!); San José del Cabo, municipio de Los Cabos, 18.I.1923, M. E. Jones 24194 (RSA-POM!); puente El Tule II, desembocadura del río El Tule, aprox. $10 \mathrm{~km}$ al SW de San José del Cabo, municipio de Los Cabos, 22 $2^{\circ} 7^{\prime} 43^{\prime \prime}$ N 10948'00" W, 29.X.2005, G. Ocampo y J. T. Columbus 1509 (RSA-POM!). 